\title{
Effect of microstructure on the thermal conductivity of disordered carbon
}

\author{
I. Suarez-Martinez and N. A. Marks \\ Nanochemistry Research Institute, Curtin University of Technology, \\ GPO Box U1987, Perth, Western Australia 6845
}

(Dated: June 2, 2011)

\begin{abstract}
Computational methods are used to control the degree of structural order in a variety of carbon materials containing primarily $\mathrm{sp}^{2}$ bonding. Room-temperature thermal conductivities are computed using non-equilibrium molecular dynamics. Our results reproduce experimental data for amorphous and glassy carbons and confirm previously proposed structural models for vitreous carbons. An atomistic model is developed for highly oriented thin films seen experimentally, with a maximum computed thermal conductivity of $35 \mathrm{Wm}^{-1} \mathrm{~K}^{-1}$. This value is much higher than that of the amorphous and glassy structures, demonstrating that the microstructure influences the thermal conductivity more strongly than the density.
\end{abstract}


Heat management is an important technological issue at multiple length scales spanning from nanoscale devices to aerospace. At the nanoscale the high density of electronic gates leads to a substantial heat load which is now a critical design factor [1]. On the macroscopic scale, aerospace and nuclear applications require materials with extreme durability as well as appropriate thermal properties [2]. Carbon structures are of interest across many of these areas due to their high melting point and a wide range of potential thermal conductivities. Highly ordered carbons such as crystalline diamond (with $\mathrm{sp}^{3}$ bonding) and carbon nanotubes (with $\mathrm{sp}^{2}$ bonding) have extremely high thermal conductivities (thousands of $\mathrm{Wm}^{-1} \mathrm{~K}^{-1}$ ) due to very long phonon mean-free paths. In these materials impurities, grain boundaries and isotopic effects control thermal transport. At the opposite end of the spectrum, amorphous carbons [3-10], glassy carbons [11] and pyrolytic chars [12] have much lower thermal conductivity, of the order of several $\mathrm{Wm}^{-1} \mathrm{~K}^{-1}$. Here the effect of local microstructure and medium-range order is paramount, with the degree of $\mathrm{sp}^{2} v s \mathrm{sp}^{3}$ bonding an important additional effect for amorphous carbons. In these systems computational studies are valuable, as they relate the microstructure of the material to the thermal behaviour. However, despite their potential usefulness, computational studies of non-crystalline carbon are scant, with almost all studies being experimental in nature, using either electrical or optical methods.

Here we apply non-equilibrium molecular dynamics (NEMD) to calculate the thermal conductivity, $\kappa$, of non-crystalline carbons spanning a wide range of densities (1.4-3.0 g/cc), coordination numbers (2.7-3.5) and microstructures. Our studies are considerably broader in scope than the only previous computational work which considered pyrolytic chars with a narrow distribution of density (1.5-1.8 g/cc) and coordination number (2.9-3.1) [13]. Our simulations were performed using the Environment Dependent Interaction Potential (EDIP) for carbon [14], a transferable potential which reproduces the structure and properties of a variety of carbon allotropes [15-17]. We relate the thermal conductivity to the microstructure, finding variations in excess of an order of magnitude, from 1 to $35 \mathrm{Wm}^{-1} \mathrm{~K}^{-1}$.

Amorphous carbon structures at different densities $(1.4-3.0 \mathrm{~g} / \mathrm{cc})$ were obtained by the liquidquench method [14] in which a randomised simple cubic lattice at a prescribed density spontaneously melts. Once the liquid was equilibrated, velocity rescaling was used to cool to room temperature in approximately 1 ps. These amorphous structures exhibit a linear relationship between $\mathrm{sp}^{3}$ fraction and density as observed experimentally [18]. The amorphous network of density $1.5 \mathrm{~g} / \mathrm{cc}$ (Fig. 1a) was subsequently annealed for $200 \mathrm{ps}$ at temperatures between 3500 and 4000K within an NVE ensemble [16]. This generated an additional set of five structures which 

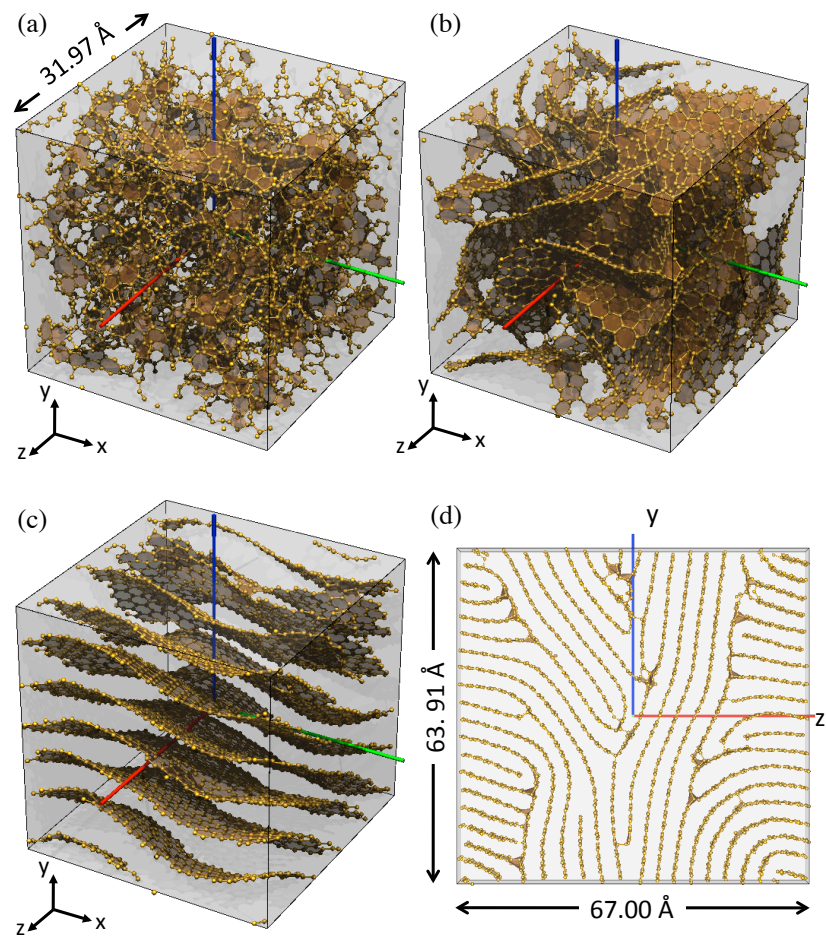

FIG. 1. Atomistic representations of the three classes of carbon material considered in this study. (a) quenched amorphous carbon with a density of $1.5 \mathrm{~g} / \mathrm{cc},(\mathrm{b}, \mathrm{c})$ structures annealed at 3500 and $4000 \mathrm{~K}$, respectively and (d) nanocrystalline oriented graphite with a density of $2.24 \mathrm{~g} / \mathrm{cc}$. The three cartesian axes are indicated: $x$ (in green), $y$ (in blue) and $z$ (in red).

were almost entirely $\mathrm{sp}^{2}$ bonded and contained graphite-like local order; two of these are shown in Fig. $1 \mathrm{~b}$ and c. A third structure type consisting of highly oriented graphite nanocrystals was generated by assembling a series of randomly rotated graphite grains all with their $c$-axis vector lying in a common plane. Realistic grain boundaries were created by annealing at $4000 \mathrm{~K}$ for $50 \mathrm{ps}$. The relaxed structure is shown in Fig. 1d. We note that graphite-like structures naturally emerge during annealing despite the absence of van der Waals attractive forces in EDIP. This ordering arises through a self-assembly process driven by $\mathrm{sp}^{3}$-to-sp ${ }^{2}$ conversion and $\pi-\pi$ repulsion which we have extensively studied in carbon onions [15, 16], nanotubes [16] and peapods [17].

The thermal conductivity, $\kappa$, was calculated using the Müller-Plathe method [19] in which a heat flux is imposed by periodically exchanging atomic velocities. The structure is divided into regular slabs along a cartesian direction and two slabs, separated by a distance of half the length of the cell, serve as hot and cold regions. The heat flux is established by swapping the velocity of the hottest atom from the cold slab, $v_{h}$, with the velocity of the coldest atom from the hot slab, $v_{c}$. 
The thermal conductivity along the relevant cartesian axis (here $x$ ) is then defined as:

$$
\kappa_{x}=-\frac{\sum_{\text {exchanges }} \frac{m}{2}\left(v_{h}^{2}-v_{c}^{2}\right)}{2 t L_{y} L_{z}<d T / d x>}
$$

where the temperature gradient $\langle d T / d x>$ is measured from the simulation by computing the average temperature of each slab over a period of time $t$ and the factor of two reflects the creation of two heat fluxes due to the periodic boundary conditions. We found that $\langle d T / d x>$ converges rapidly, typically in less than 10 ps, however our calculations were performed for at least 100 ps to ensure adequate statistics. While the frequency of velocity exchange affects $\langle d T / d x\rangle$, it has little effect on $\kappa$. In tests on a $2.5 \mathrm{~g} / \mathrm{cc}$ amorphous carbon structure the computed $\kappa$ varied over a narrow range, 2.6-2.9 $\mathrm{Wm}^{-1} \mathrm{~K}^{-1}$, for velocity exchange intervals of 3.5, 7, 14, 35 and $70 \mathrm{fs}$, corresponding to temperature differences between the hot and cold slab of 286, 192, 115, 57 and $32 \mathrm{~K}$ respectively. All subsequent results were obtained using a time interval of $35 \mathrm{fs}$. The amorphous $2.5 \mathrm{~g} / \mathrm{cc}$ system was also used to test the effect of system length [20]. Five lengths were considered, from 32.0 up to $71.9 \AA$, with little variation in $\kappa$ (less than $7 \%$ ). This behaviour is consistent with simulations of glasses [21] where the absence of crystalline order leads to scattering of phonons and relatively short mean free paths. In the amorphous models, $\kappa$ was computed by doubling the supercell depicted in Fig. 1. The resulting system size (in units of $\AA$ ) varies from $38.8 \times 38.8 \times 77.6$ at $1.4 \mathrm{~g} / \mathrm{cc}$ to $30.1 \times 30.1 \times 60.1$ at $3.0 \mathrm{~g} / \mathrm{cc}$. The largest system length effect occurs for the graphite nanocrystal model (Fig. 1d). Here, we computed $\kappa$ for lengths spanning $29.5 \AA$ up to $132.8 \AA$ finding an increase from 12 to $35 \mathrm{Wm}^{-1} \mathrm{~K}^{-1}$. Depending on the truncation point of the Taylor expansion [22], a linear extrapolation yields a bulk (infinite length) value of $71 \mathrm{Wm}^{-1} \mathrm{~K}^{-1}$, while quadratic extrapolation yields $230 \mathrm{Wm}^{-1} \mathrm{~K}^{-1}$. We therefore consider $35 \mathrm{Wm}^{-1} \mathrm{~K}^{-1}$ as a lower bound for our discussion.

One of the critical questions in the study of amorphous carbons is the dependence of the thermal conductivity on density. Experimental determination of this relationship is complicated by uncertainties in characterisation (e.g. the density and thickness of thin films) and the absence of comprehensive sets of samples. The triangles and circles in Fig. 2a illustrate the broad experimental spread, showing data from eight different authors using either the $3 \omega$ [3-5] or optical [6-10] methodology. Some of this variability is due to the specifics of sample preparation, prompting Shamsa et al. [4] to perform a series of measurements on carbon films deposited in a systematic manner. They also comment on the virtues and weakness of differing experimental approaches, a discussion we do not enter into here. By way of contrast, our computational approach (shown as 

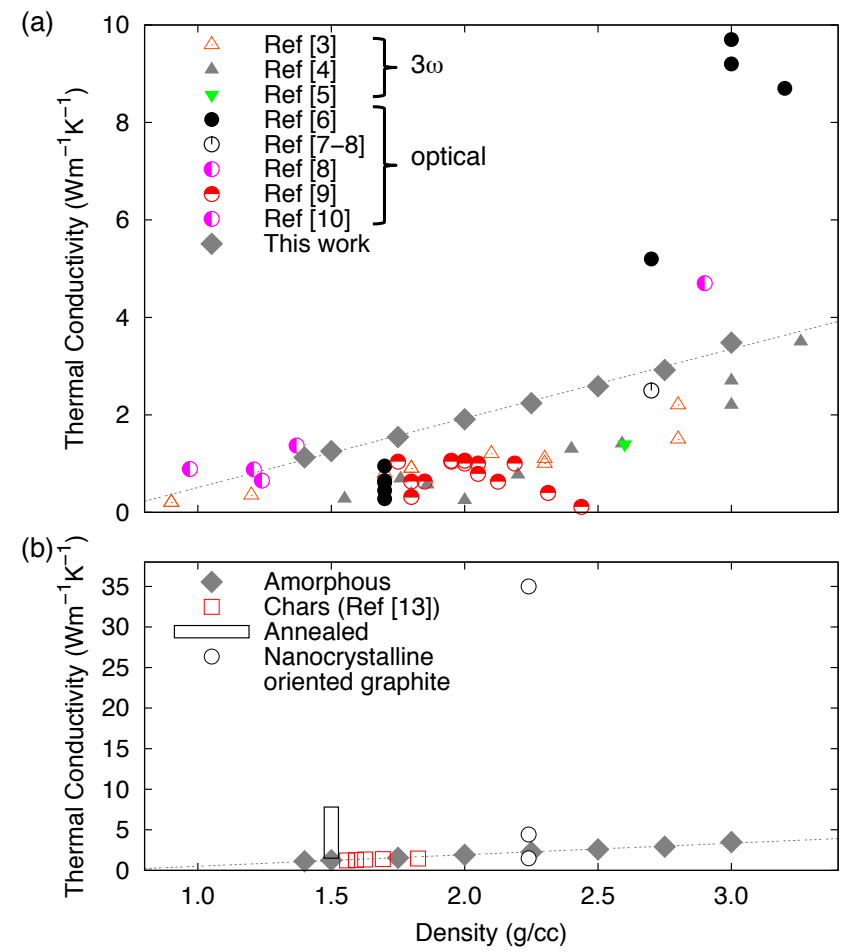

FIG. 2. (a) Room-temperature thermal conductivity of amorphous carbon structures as a function of density. Diamonds denote computational results from this work; the dotted line is a linear fit to the data. All other data are experimental measurements taken from the literature, using either resistivity $(3 \omega)$ or optical methods. (b) Thermal conductivities of four carbon materials with different microstructures. All values are determined computationally. With the exception of the chars, all data is from this work.

diamonds in Fig. 2) exhibits no such variability, finding little deviation from a straight line fitted through the simulation data points. This ability to eliminate uncertainty and identify functional relationships is a strength of the computational approach, and reminiscent of earlier work in which a linear relationship between $\mathrm{sp}^{3}$ fraction and density was demonstrated via simulation [23].

There are several lessons to be drawn from the amorphous carbon thermal conductivities in Fig. 2a. Firstly, the simulations are in good qualitative agreement with the available experimental data [3-10], particularly given the magnitude of the experimental uncertainties. Secondly, the linear dependence on density is close to that measured experimentally by Shamsa et al, [4], with the gradients differing by just $20 \%$. Thirdly, our values of the thermal conductivity are consistent with the calculations of disordered carbons by Makeev and Srivastava [13]. Using a different NEMD method [24] to that used here, they measured the thermal conductivity of a family of $\mathrm{sp}^{2}$ bonded pyrolitic chars modelled using the Brenner potential. Their networks spanned densities 
TABLE I. Hybridization fractions (in \%) and computed thermal conductivities (in $\mathrm{Wm}^{-1} \mathrm{~K}^{-1}$ ) along the three cartesian axes for the carbon structures with a density of $1.5 \mathrm{~g} / \mathrm{cc}$. The "quenched" structure was fully amorphous, while the annealed structures show increasing graphitization as quantified by the $\mathrm{sp}^{2}$ fraction.

\begin{tabular}{ccccccc}
\hline \hline $\begin{array}{c}\text { Annealing } \\
\text { temperature (K) }\end{array}$ & $\mathrm{sp}$ & $\mathrm{sp}^{2}$ & $\mathrm{sp}^{3}$ & $\kappa_{x}$ & $\kappa_{y}$ & $\kappa_{z}$ \\
\hline quenched & 31.1 & 61.2 & 7.7 & 1.2 & 1.3 & 1.3 \\
\hline 3500 & 3.1 & 90.1 & 6.8 & 3.3 & 4.0 & 2.5 \\
3625 & 3.1 & 91.9 & 5.1 & 2.8 & 5.4 & 4.6 \\
3750 & 3.1 & 92.4 & 4.5 & 2.8 & 3.5 & 1.5 \\
3875 & 3.4 & 95.1 & 1.5 & 5.2 & 4.7 & 5.0 \\
4000 & 1.5 & 98.3 & 0.2 & 7.8 & 2.3 & 3.7 \\
\hline \hline
\end{tabular}

of 1.5-1.8 g/cc and were generated by manually abstracting hydrogen atoms from a hydrocarbon precursor and quenching the resulting pure carbon network. The computed $\kappa$ of these highly $\mathrm{sp}^{2}$ networks fell between 1.25 and $1.5 \mathrm{Wm}^{-1} \mathrm{~K}^{-1}$, in very good agreement with our calculations.

In an alternative to the manual abstraction approach, we use extended annealing to generate highly $\mathrm{sp}^{2}$ networks. This method uses elevated temperatures to induce local ordering [16], allowing one to vary the $\mathrm{sp}^{2}$ fraction and average coordination in models having the same density. By virtue of the high temperatures, deep local minima are ensured. Coordinations fractions of the precursor structure and the five annealed networks are shown in Table I. The $\mathrm{sp}^{2}$ fraction increases monotonically with temperature, approaching $100 \%$ for the highest temperature of $4000 \mathrm{~K}$. Table I also reports $\kappa$ along the cartesian axis for all of the $1.5 \mathrm{~g} / \mathrm{cc}$ structures. Whereas the quenched structure has a low value of $\kappa$ and no directional dependence, the annealed structures show significantly higher values and are generally anisotropic due to the increasingly graphitic nature. The highest $\kappa$ is $7.8 \mathrm{Wm}^{-1} \mathrm{~K}^{-1}$ computed for the $4000 \mathrm{~K}$ structure (Fig. 1c) along the $x$-direction (denoted by the green line in Fig. 1d). This result can be understood given that graphite is a highly efficient thermal conductor along the basal plane and that the $4000 \mathrm{~K}$ structure shows the highest basal-plane component along the $x$-direction.

It has previously been noted that annealed carbons bear a striking resemblance to virtual porous carbon (VPC) models of glassy carbon [16]. Glassy carbons are low density carbon structures with short-range order and high $\mathrm{sp}^{2}$ fraction. Their atomistic structure is not fully understood, 
however, they are thought to be graphitic layers which bend and twist [25]. Here, we find that the computed $\kappa$ of the annealed carbons also correspond to those of experimental glassy carbons. Commercially available glassy carbon (e.g. from SPI Supplies) with densities of 1.42-1.54 g/cc and almost zero open porosity have thermal conductivity values of $4.6-6.3 \mathrm{Wm}^{-1} \mathrm{~K}^{-1}$ [26], in excellent agreement with our results. This supports the VPC model proposed by Harris [25] and confirms the predictive capability of the extended annealing approach and thermal conductivity methodology. We anticipate that a similar methodology would be equally useful to model the thermal properties of carbon foams by including large voids such as those recently reported by Tondi et al. [11] with a porosity of $96 \%$ and a thermal conductivity of $\sim 0.4 \mathrm{Wm}^{-1} \mathrm{~K}^{-1}$.

For all of the annealed structures we observed the highest $\kappa$ for the cartesian axis most aligned with the basal planes. To maximise this alignment with a single axis, we constructed the nanocrystalline oriented graphite structure depicted in Fig. 1d in which all grains have the $<001>$ vector in the same plane, i.e. the yz plane. Such a structure was recently realised experimentally in a room-temperature process using filtered cathodic vacuum arc (FCVA) deposition in conjunction with a substrate bias voltage [27]. The structure analyzed here is an atomistic representation of the conceptual diagram shown in Fig. 2 of Ref. [27]. We find that the computed $\kappa$ along the $x$-direction is very large $\left(35 \mathrm{Wm}^{-1} \mathrm{~K}^{-1}\right)$ while in the two transverse directions it is considerably less. In the FCVA experiments the carbon film grows with the $c$-axis in the biaxial plane, and hence the basal plane lies perpendicular to the substrate. Accordingly, such carbon structures will efficiently conduct heat between the film and substrate. These structures provide the prospect of a heat-removal path in semiconductor devices (avoiding the complications of nanotubes and metal contamination) or as a thermal grease when good thermal contact is required onto a rough surface.

We summarize in Fig. $2 b$ the complete computational literature for thermal conductivity of disordered carbons. Included are the three classes of structure from this study, that is, amorphous, glassy and nano-crystalline graphite. Also shown is the data of Makeev and Srivastava [13] (red boxes) whose pyrolitic chars coincide with the low density region of our amorphous structures. Varying the microstructure has an enormous effect, changing the thermal conductivity by more than an order of magnitude when the $\mathrm{sp}^{2}$ grains become highly ordered. Increasing the grain size will lead to even higher thermal conductivities, tending towards the theoretical limit for graphite of circa $4000 \mathrm{Wm}^{-1} \mathrm{~K}^{-1}$ in the basal plane [26]. This variation mimics the behaviour of highly $\mathrm{sp}^{3}$ carbons which have been extensively studied [4, 5, 28]. As shown by Angadi et al. [28] nanometre-sized $\mathrm{sp}^{3}$ grains lead to small thermal conductivities (around tens of $\mathrm{Wm}^{-1} \mathrm{~K}^{-1}$ ), while 
larger grains lead to an exponentially increasing thermal conductivity which approaches the diamond limit (2000 $\mathrm{Wm}^{-1} \mathrm{~K}^{-1}$ ). Combining our present studies of $\mathrm{sp}^{2}$ ordering with previous works on $\mathrm{sp}^{3}$ ordering provides a unified picture showing how the thermal conductivity is determined by the microstructure. We find that density plays a minor role, being important only when the structure is fully amorphous.

\section{ACKNOWLEDGMENTS}

Financial support from the Australian Research Council under grant DP0986713 is gratefully acknowledged.

[1] D. G. Cahill, W. K. Ford, K. E. Goodson, G. D. Mahan, A. Majumdar, H. J. Maris, R. Merlin, and S. R. Phillpot, J. Appl. Phys., 93, 793 (2003)

[2] K. E. Parmenter, K. Shuman, F. Milstein, C. E. Szalai, H. K. Tran, and D. J. Rasky, J. Spacecraft Rockets, 38, 231 (2001)

[3] A. J. Bullen, K. E. O’Hara, D. G. Cahill, O. Monteiro, and A. von Keudell, J. Appl. Phys., 88, 6317 (2000)

[4] M. Shamsa, W. L. Liu, A. A. Balandin, C. Casiraghi, W. I. Milne, and A. C. Ferrari, Appl. Phys. Lett., 89, $161921(2006)$

[5] A. A. Balandin, M. Shamsa, W. L. Liu, C. Casiraghi, and A. C. Ferrari, Appl. Phys. Lett., 93, 043115 (2008)

[6] C. J. Morath, H. J. Maris, J. J. Cuomo, D. L. Pappas, A. Grill, V. V. Patel, J. P. Doyle, and K. L. Saenger, J. Appl. Phys., 76, 2636 (1994)

[7] S. Falabella, D. B. Boercker, and D. M. Sanders, Thin Solid Films, 236, 82 (1993)

[8] G. Chen, P. Hui, and S. Xu, Thin Solid Films, 366, 95 (2000)

[9] W. Hurler, M. Pietralle, and A. Hammerschmidt, Diam. Relat. Mater., 4, 954 (1995)

[10] J. L. Arlein, S. E. M. Palaich, B. C. Daly, P. Subramonium, and G. A. Antonelli, J. Appl. Phys., 104, 033508 (2008)

[11] G. Tondi, V. Fierro, A. Pizzi, and A. Celzard, Carbon, 47, 1480 (2009)

[12] Subyakto, T. Hata, S. Kawai, Y. Imamura, and I. Ide, J. Wood Sci., 46, 16 (2000) 
[13] M. A. Makeev and D. Srivastava, Appl. Phys. Lett., 95, 181908 (2009)

[14] N. A. Marks, Phys. Rev. B, 63, 035401 (2001)

[15] D. W. M. Lau, D. G. McCulloch, N. A. Marks, N. R. Madsen, and A. V. Rode, Phys. Rev. B, 75, $233408(2007)$

[16] R. C. Powles, N. A. Marks, and D. W. M. Lau, Phys. Rev. B, 79, 075430 (2009)

[17] I. Suarez-Martinez, P. J. Higginbottom, and N. A. Marks, Carbon, 48, 3592 (2010)

[18] A. C. Ferrari, A. Libassi, B. Tanner, V. Stolojan, J. Yuan, L. M. Brown, S. E. Rodil, B. Kleinsorge, and J. Robertson, Phys. Rev. B, 62, 11089 (2000)

[19] F. Müller-Plathe, J. Chem. Phys., 106, 6082 (1997)

[20] P. Schelling, S. Phillpot, and P. Keblinski, Phys. Rev. B, 65, 144306 (2002)

[21] C. Oligschleger and J. Schon, Phys. Rev. B, 59, 4125 (1999)

[22] D. Sellan, E. Landry, J. Turney, A. Mcgaughey, and C. Amon, Phys. Rev. B, 81, 214305 (2010)

[23] N. A. Marks, N. C. Cooper, D. R. McKenzie, D. G. McCulloch, P. Bath, and S. P. Russo, Phys. Rev. B, 65, $075411(2002)$

[24] P. Jund and R. Jullien, Phys. Rev. B, 59, 13707 (1999)

[25] P. J. F. Harris, Philos. Mag., 84, 3159 (2004)

[26] H. Pierson, Handbook of carbon, graphite, diamond, and fullerenes: properties, processing, and applications, 1st ed. (Noyes Publications, Park Ridge, New Jersey, USA, 1993)

[27] D. W. M. Lau, D. G. McCulloch, M. B. Taylor, J. G. Partridge, D. R. McKenzie, N. A. Marks, E. H. T. Teo, and B. K. Tay, Phys. Rev. Lett., 100, 176101 (2008)

[28] M. A. Angadi, T. Watanabe, A. Bodapati, X. C. Xiao, O. Auciello, J. A. Carlisle, J. A. Eastman, P. Keblinski, P. K. Schelling, and S. R. Phillpot, J. Appl. Phys., 99, 114301 (2006) 